\title{
Research on the Mechanism of Refuting Internet Rumors in Public Health Emergencies with College Students
}

— Take Shuanghuanglian as an Example

Long Yifan ${ }^{1, \mathrm{a}}$, Wang Chunjuan ${ }^{2, \mathrm{~b}^{*}}$

\author{
${ }^{I}$ School of Marxism, Wuhan University of Technology, Wuhan, Hubei, China \\ ${ }^{2}$ School of Marxism, Wuhan University of Technology, Wuhan, Hubei, China \\ a289184@whut.edu.cn \\ b*1059577969@qq.com
}

\begin{abstract}
At the beginning of 2020, the COVID-19 epidemic broke out, and online rumors related to COVID-19 spread widely, resulting in a secondary epidemic. College students are at the center of the Internet era and are greatly affected by online rumors. Taking the "Shuanghuanglian Incident" in COVID-19 as an example, this paper summarizes the evolution process of Internet rumors in sudden public health events, analyzes the cognition status of college students on Internet rumors through empirical investigation, and deeply explores and analyzes the reasons behind them. Then put forward a reasonable, scientific and effective mechanism for college students to debunk network rumors in public health emergencies.
\end{abstract}

Keywords: Public health emergency, Internet rumors, College students

\section{突发公共卫生事件中大学生网络谣言的辟谣机制研究 - - 以双黄连事件为例}

\section{龙懿帆 ${ }^{1, a}$ 王春娟 ${ }^{2,}$ b*}

${ }^{1}$ 武汉理工大学马克思主义学院, 武汉, 湖北, 中国

2 武汉理工大学马克思主义学院, 武汉, 湖北, 中国

a289184@whut.edu.cn

b*1059577969@qq.com

\section{摘要}

2020 年初, 新型冠状肺炎疫情爆发, 与新冠相关的网络谣言泛滥, 形成次生疫情。大学生群体处于互联网时 代中心, 受到网络谣言极大的影响。本文以新冠肺炎疫情中出现的 “双黄连事件” 为例, 梳理突发性公共卫生 事件中网络谣言的演进过程, 通过实证调查分析大学生群体对网络谣言的认知状况, 并深入挖掘分析背后原因, 进而有针对性地提出突发公共卫生事件中大学生合理、科学、有效的网络谣言辟谣机制构想。

关键词：突发公共卫生事件，网络谣言，大学生

\section{1. 问题的提出}

2020 年初新冠肺炎疫情爆发, 这类突发性公共
卫生事件关系到民众的切身安全, 使其快速上升为全 社会关注的焦点。当下，正处于互联网发展大繁荣的 时期, 网络信息之繁杂、网络用户体量之大等都为网 
络谣言的滋长与传播提供了土壤, 加之突发性公共卫 生事件所具有的突发性、难以预料性，相关网络谣言 数量爆发式增长, 不仅加剧大众的焦虑情绪, 影响日 常的工作生活, 造成不必要的社会恐慌, 而且增大疫 情正常管控的难度，存在威胁国家安全的潜在风险。

其中, 大学生群体是网络用户的重要组成部分, 中国互联网信息中心发布的《第 47 次中国互联网络 发展状况统计报告》显示, 截至 2020 年 12 月, 我国 网民规模达 9.40 亿, 在我国网民职业结构中, 学生 群体最多, 占比达 $21.0 \%$ 。大学生群体作为网络信息 的受众, 部分大学生缺乏辨别能力与警惕心, 社会阅 历尚浅, 易被网络谣言所迷惑与利用, 对身心造成伤 害。本文以新型冠状肺炎疫情期间发生的各类网络谣 言为背景, 具体探究在此次舆情中网络谣言与大学生 群体的相互作用关系, 分析其背后成因, 构建突发公 共卫生事件中大学生群体网络谣言辟谣机制。

\section{2. 双黄连网络谣言演进过程}

\section{1. 网络谣言萌芽期: 主流媒体报道失范造成 误读}

2020 年 1 月 31 日晚, 人民日报官微刊登一则《最 新发现: 中成药双黄连口服液可抑制新型冠状病毒》 的报道, 报道称: “中国科学院上海药物研究所和武 汉病毒所联合研究初步发现, 中成药双黄连口服液可 抑制新型冠状病毒感染的肺炎”, 这一报道表述不够 严谨, 而报道发出时间又恰逢新冠疫情全面爆发初 期, 公众防疫意识正处于紧张时期, 引发公众高度关 注, “抑制”一词语意不明, 造成群众误读, 认为双 黄连口服液能够预防治疗新冠肺炎, 引发抢购双黄连 口服液热潮。

\section{2. 网络谣言爆发期: 社交媒体助推网络谣言 扩散传播}

藉由互联网信息传播的便利快捷开放广场式的 社交媒体, 网络谣言的传播速度极快。根据微博指数 趋势数据, 即使人民日报官微在 31 日晚近 23 时才转 载该报道引起关注, 但从 30 日到 31 日, “双黄连” 一词的微指数迅速飙升, 从 251 达到了 3511 , 环比 增长 $1298.8 \%$ 。这说明在短短的几小时里, 这一消息 就得到了指数式增长的关注量, 传播扩散速度极快。

“双黄连可抑制新型冠状病毒” 这一话题也迅速冲 上了微博热搜榜第一位, 积累了 22.2 亿的阅读量和 55.8 万的讨论量, 與论在微博上疯狂发酵。当晚, 线上药房双黄连口服液药品脱销, 线下药房门口也排 起长队。人们在微博这类开放广场式社交媒体上通过 官方媒体、网友转发等渠道了解到这一信息后, 又转 向微信、QQ 这类圈层闭环式社交媒体, 将谣言转发 给自己身边的人。这样, 网络谣言藉由从开放广场式 到圈层闭环式社交媒体的载体, 完成了从 “弱关系” 到 “强关系” 的人际传播, 形成了广泛的影响, 谣言
快速进入爆发期。

\section{3. 网络谣言消退期: 多方辟谣遏制谣言传播}

互联网时代，信息具有更强的公开性和透明性， 网络谣言往往在公开讨论中形成, 又会在大量信息流 通的过程中被多方的公开表达还原事实全貌。腾讯医 典医学团队在 1 月 31 日深夜就第一时间发布文章《辟 谣: 双黄连对新型冠状病毒有效吗?》, 对双黄连的 效用发出质疑。随着网络谣言的进一步扩散传播, 越 来越多的专业人士也开始在社交媒体进行公开辟谣。 多方意见交锋中，事实真相逐步得到还原，公众开始 质疑双黄连口服液对于治疗新型冠状病毒肺炎的效 力, 网络谣言的影响也开始减弱, 根据微博指数趋势 数据, 从 1 月 31 日到 2 月 1 日, “双黄连” 这一关 键词的微指数从 3511 迅速跌落至 612 。就在 2 月 1 日 7 时许, 人民日报官方微博便发布辟谣消息, 呼吁 群众不要抢购双黄连口服液, 至此, “双黄连可治愈 新冠” 这一网络谣言被官方正式辟谣。从时间上看, 双黄连的谣言从迅速爆发到进入消退期，仅仅不到一 天, 生命周期十分短暂。

\section{3. 双黄连网络谣言不良影响}

\section{1. 影响抗疫进程, 干扰疫情防控}

在谣言传播领域, 著名学者奥尔波特提出了一个 谣言传播公式 “ $\mathrm{R}=\mathrm{I} \times \mathrm{A}$ ” 来揭示谣言传播的基本规 律, R 代表谣言, I 代表信息的重要程度, A 代表信 息的模糊程度。新型冠状病毒肺炎疫情爆发初期。疫 情来势汹汹, 各类不实信息在网络平台上大肆传播, 真假难辨, 且这些信息与民众的生命健康安全息息相 关, 公众情绪较为紧张、惶恐, 急需一个情绪出口。 在这种突发变动情况下, 民众对信息的需求急速扩 大, 会去主动关注疫情相关信息，也更容易相信这些 信息。

关于双黄连的网络谣言, 出现在这一背景下, 迎 合了公众想要寻求一个有效治疗新型冠状病毒肺炎 方案的急切心情, 且其由官方主流媒体渠道发出, 多 家媒体转发，导致大量群众误信了该谣言，短时间内 产生了极为不良的影响: 大量人群外出至线下药店抢 购双黄连口服液, 导致居家隔离防疫措施执行不力; 出现各别患者自行服用双黄连口服液, 导致病情加重 的案例; 甚至还有线上兽用双黄连药物、双黄连月饼 也被抢购一空这种啼笑皆非的案例……双黄连谣言 对疫情防控产生了极为不利的影响。物质方面, 民众 哄抢双黄连, 经济遭受损失, 医疗资源也被浪费; 健 康方面, 双黄连口服液属于普通性病毒感冒药, 民众 自行用药可能造成身体健康受损, 疫情期间, 外出购 药增加了接触感染源的风险。精神生活方面, 这一谣 言也加剧了民众的不安情绪, 增加了疫情期间民众的 紧张心理, 长期积累, 容易形成民众的普遍恐慌心理, 不利于防疫工作开展。 


\section{2. 破坏主流媒体公信力, 影响国家政治稳定}

主流媒体是党和人民的喉舌, 承担着提供信息, 进行宣传, 整合社会, 沟通多方意见等重要功能, 地 位重要, 对维护国家政治稳定起着至关重要的作用。 而公信力是影响主流媒体发挥作用的重要因素, 所谓 公信力, 即是媒体在受众心中的信任度, 当受众信任 媒体，则会相信媒体所宣传的内容，如果媒体公信力 下降，即使宣传讲真话，受众也会怀疑。

而此次的双黄连事件, 则极大地损害了主流媒体 的公信力。“双黄连口服液可治愈新型冠状病毒肺炎” 这一网络谣言的起因, 就是人民日报官方微博一条表 述不够科学准确的报道引起了公众的错误解读。而其 作为主流媒体的较高的公信力和较强权威性, 使得这 一错误的信息没有及时得到质疑, 反而让更多存疑的 民众相信了这一网络谣言。而就在这一谣言喧器尘上 时, 人民日报第二天清晨又发布与其之前报道相悖的 辟谣提醒, 这在民众眼中, 无疑是 “反转打脸” 的新 闻, 这时, 公众的关切焦点已经从辨别谣言真伪转移 到了质疑主流媒体的媒介素养上来, 而相关公司闻声 暴涨的股价, 更让许多怀疑 “利益输送”、“广告新 闻” 的阴谋论大行其道, 这些都对我国主流媒体的公 信力造成了极大的损害。一旦公众开始质疑主流媒体 是否还代表着国家和人民的利益, 主流媒体的宣传效 果就会大打折扣, 其公信力也会大大下降。这破坏了 公众的政治信任，极不利于国家的政治稳定。

\section{4. 大学生群体对网络谣言的认知和应对}

\section{1. 大学生群体网络谣言接触渠道}

当代大学生作为成长在互联网发展背景下的 “ $Z$ 世代”, 浸淫在网络和电子产品所形成的虚拟空间中, 其日常生活基本离不开网络。根据线上调查结果, 有 $55 \%$ 的大学生每日平均上网时间约达到 5 小时以上。 活跃于微博、微信公众号上的自媒体、QQ、微信等即 时聊天工具是大学生在网上获取信息的最主要渠道, 多达 $89.38 \%$ 的同学选择了这一选项作为自己 “网上 获取信息的主要渠道”。这些渠道, 也是双黄连事件 中网络谣言迅速扩散传播的媒介载体, 在线下的访谈 中, 多名学生的回答也说明了这一点: “第一次看到 双黄连口服液和新冠的网络谣言, 是在微博热搜上”、

“我是在刷微博时看到了人民日报官博发的消息”。 结合线上问卷调查和线下访谈来看, 网络社交媒体已 经成为大学生群体接触网络信息的主要渠道, 此次双 黄连與情事件中, 大部分学生藉由网络社交媒体接触 到相关网络谣言。

\section{2. 大学生群体网络谣言应对行为}

在與情中, 网络个体对网络谣言的应对行为主要 包括判断辨别、传播和辟谣。在谣言被认定之前, 个 体主要对疑似网络谣言的信息进行判断、分辨和传
播; 在网络谣言被明确认定为后, 个体主要的应对行 为主要为是否主动进行辟谣。

在双黄连與情中, 在相关网络谣言被明确认定之 前, 也就是网络谣言的爆发期, 大学生群体对网络谣 言的判断辨别能力较弱。根据线上调查, 关于 “在这 次疫情中, 您是否有被疫情相关网络谣言 (如双黄连 可治愈新冠等)欺骗的经历”这一问题,有多达 $26.25 \%$ 的学生选择了 “有过被欺骗的经历”。在线下访谈调 查中, 数名被调查的学生中也有 2 名坦言辟谣之前, 相信了双黄连能治愈新冠。可见, 虽然大学生群体受 过高等教育, 有较好的科学素养, 但由于其独立思考 的能力较为溃乏, 容易依赖轻信外界权威信息, 受骗 的人不在少数。而在被问到, 该消息尚未得到确认时, 是否传播过这一讯息, 参与访谈的同学无一例外地表 示和身边的同学、家人传播分享过, 且多是通过即时 通信 APP 传播。这说明习惯使用网络社交媒体的大学 生们对于新奇且重要的网络信息的传播倾向极高, 无 形之中助推了网络谣言的扩散。

在双黄连网络谣言正式辟谣后, 大学生群体较少 主动进行辟谣。在 “当您遇到一则您认为是谣言的网 络消息时, 您会采取什么样的行动” 这一问题中, 有 多达 $26.25 \%$ 的学生选择了 “不采取任何行动”。在 线下访谈的同学中, 则只有一名同学表示在辟谣后及 时提醒了家人不要去抢购双黄连。可见大学生群体网 络主体的责任意识不高, 较少进行主动辟谣, 这不利 于抑制网络谣言的传播。

\section{3. 网络谣言对大学生群体的政治信任造成 冲击}

“谣言与政治相伴而生, 天然具有政治属性。” 相关研究显示, 网络谣言对大学生影响不仅仅停留在 简单的误信欺骗层面, 还会影响大学生群体的政治信 任。在双黄连事件中, 两家主流媒体虽然在第二日对 自身发布的内容进行了解释说明, 修正了工作中的错 误。但由于其报道失范的问题直接造成谣言的产生, 并且未在谣言爆发期及时进行正确引导。这无疑降低 了其公信力, 影响民众对于政府的信任, 容易造成“塔 西佗陷阱”。

这一问题在大学生群体中尤为明显, 在问卷调查 中, 关于 “面对各种来源不明消息的冲击 (如双黄连 事件）, 部分政府部门应对不当的情况, 您觉得您是 否陷入了塔西佗陷阱” 这一问题, 有多达 $63.76 \%$ 的 学生选择了 “有时候会” 和 “完全陷入”。线下访谈 中, 也有许多学生表达了在这一與情中对主流媒体的 失望一一学生 W 谈到, “在我一直以来的印象里, 这 类代表着国家和政府的主流媒体是十分具有权威性 的, 当消息出来时, 出于对这类权威媒体的信任, 我 觉得可信度很大。结果第二天反转了, 我特别失望, 感觉这么大的媒体怎么能闹着玩一样。” 学生 C 则说

“我从始至终就没有相信双黄连能治愈新冠, 因为我 的生活常识告诉我一种新的疾病没有那么容易找到 
有效药物。而且疫情期间经常有各种谣言流传, 所以 网络上的消息我觉得不一定可信。看到是人民日报发 的, 还是挺震惊的, 我觉得这类官方媒体不能像追逐 流量的营销号一样发这种不靠谱的消息。”可见，双 黄连與情中，官方媒体的把关失范，极大地削弱了政 府的公信力, 对大学生群体的政治信任造成冲击, 使 得不少大学生陷入塔西佗陷阱, 容易产生消极的社会 心态，危害其身心健康。

\section{5. 突发公共卫生事件中大学生群体网络谣言 辟谣机制}

\section{1. 辟谣核心一一明晰的谣言认定机制}

谣言的认定机制, 是辟谣的核心机制。抑制谣言 的传播, 消解谣言带来的不良影响, 最有效的方式就 是向公众指出谣言的谬误之处。在前文关于这次與情 网络谣言生命周期的分析中, 不难发现, 当第二天双 黄连相关消息被人民日报辟谣后, 其关注度迅速下 降。可见, 信息一旦被认定为谣言, 其传播力和影响 力就会迅速下降。要建立高效的网络谣言辟谣机制, 就要建立起一个明晰的谣言认定机制, 提高辟谣的速 度, 减轻谣言的引发的影响。

当前, 网络谣言的认定机制主要有官方核查、专 家科普、本人辟谣、采访求证等。谣言的认定方式较 为多样, 涉及多个部门, 與情较为复杂, 一旦发生反 转, 就会损害权威部门的公信力。因此, 需要建立一 个权威辟谣平台来认定谣言, 联合多方资源, 整合更 多的信源求证, 用事实和科学辟谣, 及时发布辟谣信 息，提高公信力。

\section{2. 辟谣主体一一主流媒体做好把关人角色, 多方协同治理}

在信息飞速流通的时代，媒体力量是辟谣的主力 军, 在这个信息纷杂, 众说纷纭的时代, 主流媒体作 为有权威性的官方发声渠道, 格外受到群众信任。在 突发公共事件中, 网络谣言泛滥, 主流媒体应当主动 担当起辟谣主体的责任, 及时发布辟谣信息, 缓解䁷 情中公众的紧张情绪, 引导與论导向。同时, 主流媒 体作为权威的发声渠道, 应做好 “把关人” 的职责, 加强对信息的审核, 审慎对待将要发布的报道, 做好 信息的求证考察, 及时对自身工作进行自纠自查, 避 免发生多次反转，损害自身的公信力。

同时, 在这次事件中谣言的产生和扩散的首要原 因, 就是谣言萌芽期主流媒体的报道失范。这说明互 联网时代网络各主体对于主流媒体具有较大的依赖 性。这种依赖使得公众较为盲目地相信权威, 缺少多 元信源, 会损害公众面对信息的辨别能力。这种情况 下, 应该引入多方协同治理机制, 积极鼓励网络多元 主体发声辟谣, 引导多方媒介力量主动表达求证, 在 信息的流通讨论中使其自发被补全, 消解网络谣言的
影响力, 形成一个多方主体联动辟谣的网络发声机 制，使谣言迅速消解。

\section{3. 辟谣关键一一培养大学生群体公民意识, 增强谣言免疫力}

构建突发公共事件中大学生群体网络辟谣机制 的关键, 要培育大学生群体的公民意识, 增强大学生 群体的谣言免疫力。大学生群体处于情绪较为冲动的 年龄阶段, 关心社会公共事务, 虽然有较好的科学素 养, 但社会阅历尚浅, 心态较为脆弱, 容易受到网络 谣言的煽动影响。反复的谣言舆情, 会影响大学生群 体的政治信任水平, 使得他们容易陷入塔西佗陷阱, 不利于国家政治安全。要发挥高校思政教育工作的思 想引领作用, 培育大学生群体良好的公民意识, 引导 大学生积极表达, 增强网络主体的责任意识, 以理性 态度面对复杂思想和谣言的冲击, 提高其对网络谣言 的辨别能力, 积极主动承担辟谣责任, 减少谣言传播, 增强其谣言免疫力。

\section{6. 结论}

“双黄连” 事件中，网络谣言对大学生群体影响 不仅仅停留在简单欺骗层面, 因为舆情的反转, 更削 弱了其对于官方主流媒体的信任，使其陷入塔西佗陷 阱。这更是反映出大学生群体对于主流媒体的依赖和 思想的脆弱性。因此，构建突发公共事件中大学生网 络谣言辟谣机制的关键, 是要增强其对网络谣言的免 疫力。同时, 要建立明晰的谣言认定机制, 主流媒体 做好把关工作，引入多方协同治理，强化多元主体的 发声辟谣意识, 形成一个认定明晰, 主体多元的科学 网络谣言治理机制。

\section{项目基金}

本文为国家级大学生创新创业训练计划资助《重 大公共事件中大学生对网络谣言的认知、传播和引导 机制研究——以 2020 新冠疫情为例》 （S202010497140）的阶段性成果之一。

\section{REFERENCES}

[1] Peng Lan. (2017) Introduction to network communication. China Renmin University Press, Peking.

[3] Guo Xiaoan. (2015) A study on the social psychology of Internet rumors in contemporary China. China Social Sciences Publishing House, Peking.

[4] Chang Yanmin. (2020) Research on the network rumor governance system under the guidance of political trust cultivation. Ph. D thesis, Zhengzhou University. 
[5] Tian Shuo. (2021) The rumor propagation and governance of "Shuanghuanglian Incident" from the perspective of social governance. Journal of News Research, 12(01): 23-25.

[6] Xiang Qingping. Lei Yuejie. (2020) Research on online rumor spreading and refuting mechanism of public health emergencies. Journal of Western, 19: $27-34$.

[7] Luo Yuting, Luo Hong. (2020) On the improvement of college students' ability to recognize online rumors. Ideological \& Theoretical Education, 03: 73-79. 